\title{
AN ARC AS THE INVERSE LIMIT OF A SINGLE NOWHERE STRICTLY MONOTONE BONDING MAP ON [0,1]
}

\author{
J. W. ROGERS, JR.
}

Introduction. It is well known that a nondegenerate continuum (compact, connected metric space) is chainable if and only if it is homeomorphic to the limit of an inverse sequence of arcs with bonding maps onto. Several authors have studied chainable continua as inverse limits of arcs and in particular Henderson [3] and Mahavier [4] have contributed to the problem of characterizing the class $C$ of chainable continua that are homeomorphic to the inverse limit of arcs using a single bonding map. Henderson showed that the pseudoarc is a member of $C$ and Mahavier gave some examples of chainable continua not in $C$ but proved that each chainable continuum is embedded in some member of $C$.

In the inverse limit description of a chainable continuum the bonding maps mimic the pattern (see Bing [1] for terminology related to chains) of the chains. Thus, one expects (proved by Capel [2]) an inverse limit with monotone bonding maps to be an arc where a monotone map corresponds to a chain going straight through another chain. Likewise the crooked chains that yield the pseudo-arc give rise to maps with corresponding "crooked" graphs. Thus, the question arises as to what kind of maps will yield an arc or more specifically what kind of single bonding map will yield an arc? In particular, is it possible for an arc to be the inverse limit of a nowhere strictly monotone map on $[0,1]$ ? As the above title indicates, the answer is yes.

The author is grateful to the referee for suggestions regarding the proof of Theorem 1.

Preliminaries. A continuous function $f$ on the interval $[0,1]$ is rising [falling] at $x$ if and only if $x \neq 1, x$ is in $[0,1]$, and there is a segment $s$ containing $x$ such that (a) if $x=0$, then $f(y)>f(0)$ $[f(y)<f(0)]$ for every number $y$ in both $s$ and $[0,1]$ and (b) if $x>0$, and $u$ and $v$ are numbers in $s$ and in $[0,1]$ such that $u<x<v$, then $f(u)<f(x)<f(v)[f(u)>f(x)>f(v)]$. The function $f$ is nowhere strictly monotone if it is neither rising nor falling at any number $x$.

Let $I$ denote the interval $[0,1]$. Suppose $f$ is a continuous function from $I$ onto $I$ and $\lim f$ is the inverse limit space with $f$ as a single bonding map, i.e., $\lim f$ is the inverse limit space for the inverse limit sequence $I \stackrel{f}{\leftarrow} I f I \cdots$. It is known that if $f$ is monotone, then $\lim f$

Received by the editors February 27, 1967. 
is an arc. W. S. Mahavier told the author that if $f(x)<x(0<x<1)$, then $\lim f$ is an arc so long as $f$ is monotone on some interval $[z, 1]$, where $0<z<1$. Theorem 1 may be regarded as a generalization of this result. The examples show that $f$ may be very far from monotone, and yet $\lim f$ still be an arc.

Lemma 1. Suppose $f$ is a continuous function from $[0,1]$ onto $[0,1]$ such that $f(0)=0$ and there is a number $z(0<z<1)$ such that if $0<x \leqq z$, $f(x)<x$. Then if $X\left(x_{1}, x_{2}, \cdots\right)$ is a point of $\lim f$ distinct from $(0,0,0, \cdots)$, there exists a positive integer $n$ such that (1) $z \leqq x_{n},(2)$ if $i$ is a positive integer less than $n$, then $x_{i}>z$, and (3) if $i$ is a positive integer greater than $n$, then $x_{i}<z$.

(The proof is omitted.)

TheOREM 1. Suppose $f$ and $g$ are continuous functions from $[0,1]$ onto $[0,1]$ such that $f(0)=g(0)=0$, and such that there is a number $z(0<z<1)$ such that (1) if $0<x \leqq z, f(x)<x$ and $g(x)<x$, and (2) if $z \leqq x \leqq 1, f(x)=g(x)$. Then $\lim f$ and $\lim g$ are topologically equivalent.

Proof. For each point $X\left(x_{1}, x_{2}, \cdots\right)$ of $\lim f$ or of $\lim g$ distinct from $(0,0,0, \cdots)$, let $N(X)$ denote the positive integer $n$ associated with $X$ as in the statement of Lemma 1. Let $T$ denote the transformation whose domain is $\lim f$ such that $T(0,0,0, \cdots)=(0,0,0, \cdots)$, and if $X\left(x_{1}, x_{2}, \cdots\right)$ is a point of $\lim f$ distinct from $(0,0,0, \cdots)$, then $T(X)=\left(g^{n}\left(x_{n+1}\right), g^{n}\left(x_{n+2}\right), \cdots\right)$, where $n=N(X)-1$. We show that $T$ is a homeomorphism from $\lim f$ onto $\lim g$.

It is easy to verify that $T$ is one-to-one from $\lim f$ onto $\lim g$.

To show that $T$ is continuous, take points $X\left(x_{1}, x_{2}, \cdots\right)$ in $\lim f$ and $X^{\prime}\left(x_{1}^{\prime}, x_{2}^{\prime}, \cdots\right)=T(X)$ in $\lim g$ and an open set $R^{\prime}$ in lim $g$ containing $X^{\prime}$. We may assume that for some $m$ and some open set $Q$ in $[0,1]$ containing $x_{m}, R^{\prime}$ is the set of all points of $\lim g$ whose $m$ th coordinate is in $Q$. If $X \neq(0,0, \cdots)$ we may also assume that $m>N(X)$ and $Q$ is open in $(z, 1]$. Furthermore, if $R$ is the set of all points in $\lim f$ whose $m$ th coordinate is in $Q$, then $T(R)$ lies in $R^{\prime}$; indeed, $T(R)=R^{\prime}$.

If $X=(0,0, \cdots)$, there is a number $w(0<w<1)$ such that if $\left(y_{1}, y_{2}, \cdots\right)$ is a point of the complement $C\left(R^{\prime}\right)$ of $R^{\prime}$ in $\lim g$, then $y_{m} \geqq w$. There is a positive integer $j>m$ such that if $\left(y_{1}, y_{2}, \cdots\right)$ is in $C\left(R^{\prime}\right)$, then $y_{j}>z$. The set $R$ of all points of $\lim f$ whose $j$ th coordinate is in the set $[0, z)$ is open in $\lim f$ and contains $X$; and $T(R)$ lies in $R^{\prime}$.

Since $\lim f$ is compact, $T$ is a homeomorphism.

Lemma 2. Suppose $A$ and $B$ are two points of the compact (metric) 
continuum $M$, and if $P$ is a point of $M$ distinct from $A$ and $B$, and $R$ is a region containing $P$, then $R$ contains a subcontinuum of $M$ that contains $P$ and separates $A$ from $B$. Then $M$ is an arc from $A$ to $B$.

(The proof is omitted.)

Examples. Here we construct an example of a continuous function $g$ from $[0,1]$ onto $[0,1]$ which is nowhere strictly monotone, such that $g(0)=0, g(1)=1$, and if $0<x<1$, then $g(x)<x$, but such that $\lim g$ is an arc. The function $g$, however, is constant on the interval $\left[0, \frac{1}{2}\right]$, so (making use of Theorem 1 ) we construct a function $f$, having all the above properties, which is not constant on any interval.

In the following, the word box means a rectangular disk in the plane with sides parallel to the axes. The phrase chain of boxes will be used to refer to certain collections of boxes which resemble chains.

Suppose $M$ is a subset of $[0,1] \times[0,1]$, and $D=\left[x_{1}, x_{2}\right] \times\left[y_{1}, y_{2}\right]$ is a rectangular disk in the plane. Then the copy of $M$ in $D$ (denoted by $C(M, D))$ is the set of points $\left(x^{\prime}, y^{\prime}\right)$, where for some point $(x, y)$ of $M x=\left(x^{\prime}-x_{1}\right) /\left(x_{2}-x_{1}\right)$ and $y=\left(y^{\prime}-y_{1}\right) /\left(y_{2}-y_{1}\right)$. If $W$ is a collection of subsets of $[0,1] \times[0,1], C(W, D)$ denotes the collection of all copies of elements of $W$ in $D$.

Let $g_{1}, g_{2}$, and $g_{3}$ denote functions from $[0,1]$ onto $[0,1]$ such that $g_{1}(0)=g_{1}(2 / 3)=0, g_{1}(1 / 3)=g_{1}(1)=1$, and $g_{1}$ is linear on each of the intervals $[0,1 / 3],[1 / 3,2 / 3],[2 / 3,1] ; g_{2}(x)=g_{1}(1-x)$; and $g_{3}(x)=x$. If $i=1,2$, or 3 , and $n$ is a positive integer, let $W_{i}(n)$ denote the chain of $3^{n+1}$ boxes lying in $[0,1] \times[0,1]$, each of which has as opposite vertices the points $\left(t_{j-1}, g_{i}\left(t_{j-1}\right)\right)$ and $\left(t_{j}, g_{i}\left(t_{j}\right)\right)$ for some positive integer $j, 1 \leqq j \leqq 3^{n+1}$, where for each nonnegative integer $j, t_{j}=j(1 / 3)^{n+1}$. The chain $W_{1}\left(W_{2}\right)$ will be said to be ascending (descending) at each of its boxes (or links) whose projection lies in one of the intervals $[0,1 / 3]$ and $[2 / 3,1]$, and descending (ascending) at each of its links whose projection lies in the interval $[1 / 3,2 / 3] . W_{3}$ is ascending at each of its links. A similar terminology will be used for copies of $W_{1}$, $W_{2}$, and $W_{3}$ and for other chains when appropriate.

Let $a_{0}, a_{1}, \cdots$ denote a sequence of numbers such that $a_{0}=0$, and if $n$ is a positive integer, $a_{n}=1-2^{-n}$. For each positive integer $i$, let $B_{i}=\left[a_{i}, a_{i+1}\right] \times\left[a_{i-1}, a_{i}\right]$. Let $C_{0}$ denote the chain of boxes $B_{1}$, $B_{2}, \cdots$. For each positive integer $n$, let $C_{n}$ denote the chain of boxes to which the box $B$ belongs if and only if for some positive integer $i, B$ lies in $B_{i}$ and in some box $B^{\prime}$ of $C_{n-1}$ such that (1) if $i \leqq n, B$ is an element of the copy in $B^{\prime}$ of $W_{1}(i)\left[W_{2}(i)\right]$ if $C_{n-1}$ is ascending [descending] at $B^{\prime}$, and (2) if $i>n, B$ is an element of the copy in $B^{\prime}$ of $W_{3}(n)$. Roughly, the chain $C_{n}$ "wiggles through" all the boxes of $C_{n-1}$ 
that are in $B_{1}, B_{2}, \cdots, B_{n}$, then goes "straight through" the rest of the boxes in $C_{n-1}$.

Let $G$ denote the simple graph which consists of the point $(1,1)$ and all the points on the $x$-axis with abscissas $0, \frac{1}{2}$, or between, together with all the points common to the point sets $C_{1}{ }^{*}, C_{2}{ }^{*}, \cdots$, where $C_{n}{ }^{*}$ denotes the sum of the elements of $C_{n}$. Let $g$ denote the function from $[0,1]$ onto $[0,1]$ whose graph is $G$. Then $g$ is continuous and nowhere strictly monotone.

We now show that $\lim g$ is an arc, using Lemma 2, i.e., we show that if $A$ and $B$ denote the points $(0,0,0, \cdots)$ and $(1,1,1, \cdots)$, respectively, of $\lim g$, and $\epsilon>0$, and $X\left(x_{1}, x_{2}, \cdots\right)$ is a point of $\lim g$ distinct from $A$ and from $B$, then there is a subcontinuum of $\lim g$ containing $X$, of diameter less than $\epsilon$, which separates $A$ from $B$. (We use the usual metric: if $X\left(x_{1}, x_{2}, \cdots\right)$ and $Y\left(y_{1}, y_{2}, \cdots\right)$ are points of $\lim g, d(X, Y)=\sum_{i=1}\left|x_{i}-y_{i}\right| \cdot 2^{-i}$. $)$

Suppose $\epsilon>0$ and $X\left(x_{1}, x_{2}, \cdots\right)$ is a point of $\lim g$ distinct from $A$ and $B$. Let $n$ denote a positive integer such that $(1 / 3)^{n}<\epsilon$. For each positive integer $i$, let $P_{i}$ denote the point $\left(x_{i+1}, x_{i}\right)$ of the graph $G$. There exists an infinite sequence of point sets $Q_{1}, Q_{2}, \cdots$ such that for each $i$, (1) $Q_{i}$ is either the origin, a subinterval of $[0,1 / 2]$, or some box of $C_{n}$, (2) $P_{i}$ lies in $Q_{i}$, and (3) the domain of $Q_{i}$ is the range of $Q_{i+1}$. Thus, if for each positive integer $i, R_{i}$ denotes the range of $Q_{i}$ and $f_{i}$ denotes $g \mid R_{i+1}$, we have an inverse limit system $R_{1} f_{\leftarrow} R_{2} f_{2}$ $R_{3 \leftarrow} \stackrel{f_{3}}{\leftarrow}$. such that for each $i$, (1) $R_{i}$ is either a point or an interval of length $\leqq \frac{1}{2}(1 / 3)^{n}$ (since none of the boxes of $C_{n}$ are taller than those in $B_{1}$, which have height $\left.\frac{1}{2}(1 / 3)^{n}\right)$, and (2) $f_{i}$ throws $R_{i+1}$ onto $R_{i}$. The inverse limit space $M$ thus obtained is a subcontinuum of $\lim g$ containing $X$, of diameter $\leqq \frac{1}{2}(1 / 3)^{n}[1 / 2+1 / 4+1 / 8+\cdots]=\frac{1}{2}(1 / 3)^{n}$ $<\epsilon$.

To show that $M$ separates $A$ from $B$, let $z$ denote some number in the interior of the range, $R_{j}$, of $Q_{j}$, where $j>n$. Then the set $K$ of all points of $\lim g$ not in $M$ is divided into the mutually separated point sets $M_{A}$ and $M_{B}$, consisting respectively of (1) all points of $K$ whose $j$ th coordinates are less than $z$ and (2) all points of $K$ whose $j$ th coordinates are greater than $z$.

Thus the hypothesis of Theorem 2 is satisfied, and consequently $\lim g$ is an arc.

Now, $g$ is constant on the interval $[0,1 / 2]$. We obtain as follows a function $f$ having properties similar to $g$, but which is not constant on any interval. There exists a simple graph $H$ in $[0,1] \times[0,1]$ containing the points $(0,0),(1 / 2,1)$, and $(1,0)$ such that the common part of the graph $G$ and the rectangle $[1 / 2,2 / 3] \times[0,1 / 2]$ is a copy in 
$[1 / 2,2 / 3] \times[0,1 / 2]$ of $H$. For each $n$, let $D_{n}$ denote the box $\left[2^{-n-1}, 2^{-n}\right]$ $\times\left[0,2^{-n-1}\right]$. Let $F$ denote the simple graph which consists of the origin and all the points of $G$ with abscissas $1 / 2,1$, or between, together with all the points $P$ such that for some $n, P$ is a point of the copy of $H$ in $D_{n}$. Let $f$ denote the function from $[0,1]$ onto $[0,1]$ whose graph is $F$. By virtue of Theorem 1 , since $\lim g$ is an $\operatorname{arc}, \lim f$ is an arc. Moreover, $f$ is nowhere strictly monotone, and is not constant on any interval.

\section{REFERENCES}

1. R. H. Bing, Snake-like continua, Duke Math. J. 18 (1951), 653-663.

2. C. E. Capel, Inverse limit spaces, Duke Math. J. 21 (1954), 233-245.

3. G. W. Henderson, The pseudo-arc as an inverse limit with one binding map, Duke Math. J. 31 (1964), 421-426.

4. W. S. Mahavier, A chainable continuum not homeomorphic to an inverse limit on $[0,1]$ with only one bonding map, Proc. Amer. Math. Soc. 18 (1967), 284-286.

EMORY UNIVERSITY 